\title{
Microbubble-mediated sonothrombolysis with BR38 of a venous full blood thrombus in a rat embolic stroke model
}

\author{
Tobias Braun ${ }^{1,2 \#}$, Laura Sünner ${ }^{2 \#}$, Maaike Hachenberger ${ }^{1,2}$, Clemens Müller $^{3}$, Astrid Wietelmann ${ }^{4}$, \\ Martin Juenemann ${ }^{1,2}$, Jörn Pons-Kühnemann ${ }^{5}$, Manfred Kaps ${ }^{1}$, Tibo Gerriets ${ }^{1,2,6}$, Marlene Tschernatsch ${ }^{1,2,6}$, \\ Joachim Roth ${ }^{7}$, Mesut Yenigün ${ }^{1,2}$
}

${ }^{1}$ Department of Neurology, Faculty of Medicine, Justus-Liebig-University, Giessen, Germany; ${ }^{2}$ Heart \& Brain Research Group, Justus-LiebigUniversity Giessen and Kerckhoff Clinic, Bad Nauheim, Germany; ${ }^{3}$ Department of Radiology, Kerckhoff Clinic, Bad Nauheim, Germany; ${ }^{4}$ Scientific Service Group Magnetic Resonance Imaging, Max-Planck-Institute for Heart and Lung Research, Bad Nauheim, Germany; ${ }^{5}$ Institute of Medical Informatics, Department of Medical Statistics, Justus-Liebig-University, Giessen, Germany; ${ }^{6}$ Department of Neurology, Gesundheitszentrum Wetterau, Bad Nauheim, Germany; ${ }^{7}$ Department of Veterinarian Physiology and Biochemistry, Justus-Liebig-University, Giessen, Germany

Contributions: (I) Conception and design: T Braun, M Yenigün, T Gerriets; (II) Administrative support: M Kaps, T Gerriets, A Wietelmann, C Müller; (III) Provision of study materials or patients: A Wietelmann, C Müller; (IV) Collection and assembly of data: L Sünner, M Tschernatsch, M Hachenberger, M Juenemann; (V) Data analysis and interpretation: T Braun, L Sünner, J Pons-Kühnemann, M Yenigün, J Roth; (VI) Manuscript writing: All authors; (VII) Final approval of manuscript: All authors.

\#These authors contributed equally to this work.

Correspondence to: Tobias Braun, MD. Department of Neurology, Justus-Liebig-University, Klinikstraße 33, D-35385 Giessen, Germany. Email: tobias.braun@neuro.med.uni-giessen.de.

Background: Early recanalization of an occluded vessel is associated with a better clinical outcome in acute ischemic stroke. Intravenous thrombolysis using recombinant tissue plasminogen activator (rt-PA) is only available in a minority of patients and often fails to reopen the occluded vessel. Mechanical recanalization is more effective in this matter but only available for selected patients when a thrombectomy centre can be reached. Therefore, sonothrombolysis might represent an alternative or complementary approach. Here, we tested microbubble-mediated sonothrombolysis (mmSTL) in a thromboembolic stroke model for middle cerebral artery occlusion (MCAO) in rats.

Methods: Sixty-seven male Wistar rats underwent MCAO using an autologous full blood thrombus and were randomly assigned to four groups receiving rt-PA, mmSTL, a combination of both, or a placebo. Diagnostic workup included neurological examination, assessment of infarct size, and presence of intracerebral haemorrhage by magnetic resonance imaging (MRI) and presence of microbleedings in histological staining.

Results: Neurological examination revealed no differences between the treatment groups. In all treatment groups, there was a reduction in infarct size 24 hours after MCAO as compared to the placebo $(\mathrm{P} \leq 0.05)$, but there were no differences between the active treatment groups $(\mathrm{P}>0.05)$ (placebo $0.75 \pm 0.10 \mathrm{~cm}^{3} ; \mathrm{mmSTL}$ $0.43 \pm 0.07 \mathrm{~cm}^{3} ; \mathrm{rt}-\mathrm{PA} 0.4 \pm 0.07 \mathrm{~cm}^{3} ; \mathrm{mmSTL}+\mathrm{rt}-\mathrm{PA} 0.27 \pm 0.08 \mathrm{~cm}^{3}$ ). Histological staining displayed intracerebral microbleedings in all animals. The frequency of gross bleeding detected by MRI did not differ between the groups (placebo 3; mmSTL 4; rt-PA 2; mmSTL + rt-PA 2; P>0.05) and was not associated with worse performance in clinical testing $(\mathrm{P}>0.05)$. There were no statistical differences in the mortality between the groups $(\mathrm{P}>0.05)$.

Conclusions: Our study showed the efficacy and safety of mmSTL with or without rt-PA in an embolic rat stroke model using a continuous full blood thrombus. Sonothrombolysis might be useful for patients who need to be transported to a thrombectomy centre or for those with distal vessel occlusion.

^ ORCID: 0000-0003-1738-2773. 
Keywords: Embolic stroke; microbubbles; rat; sonothrombolysis; ultrasound (US)

Submitted Feb 26, 2021. Accepted for publication Apr 14, 2021.

doi: $10.21037 /$ atm-21-75

View this article at: http://dx.doi.org/10.21037/atm-21-75

\section{Introduction}

Stroke is the second cause of death and the leading cause of disability in the world $(1,2)$. The majority of ischemic strokes is caused by cerebral embolism (3).

Achieving recanalization in a timely manner is a key goal in developing better stroke thrombolytic therapy. This is of importance in patients who are not eligible for recanalization therapy [intravenous recombinant tissue plasminogen activator (rt-PA) or thrombectomy] or to bridge time in patients with or without rt-PA treatment who need to be shipped from a remote hospital to a thrombectomy centre. In particular, patients with occlusion of smaller vessels need a reliable, effective, and safe therapy that can be performed in all stroke treating hospitals, as such a treatment is currently lacking.

Currently, the only approved thrombolytic treatment for acute ischemic stroke is rt-PA delivered intravenously within 4.5 hours of stroke onset (4). Less than $10 \%$ of all ischemic stroke patients are eligible for therapy, even though current research has suggested a higher rate when using elaborated imaging [perfusion imaging in computed tomography (CT) and magnetic resonance imaging (MRI)] for patient selection (5-8). However, only a small number of patients can be treated with rt-PA because there are several contraindications apart of the 4.5-hour "time window", such as therapeutic anticoagulation, prior surgery, or active cancerous diseases. In Germany, $16.4 \%$ of patients admitted for ischemic stroke receive rt-PA treatment (9). However, rt-PA achieves successful recanalization in less than half of those treated (10). In patients with large vessel occlusion, recanalization can only be achieved in $10 \%$ of the patients using rt-PA (11). For these reasons, rt-PA treatment seems to have reached its limits. As recanalization is the most important factor for a satisfying patient outcome (12), there is dire need for a therapy that can achieve recanalization of an occluded vessel in a timely manner.

A complementary approach is catheter-based mechanical recanalization. This emerging treatment is gaining in importance and is associated with a satisfying patient outcome (13). Again, this approach is only possible in a minority of patients because it is currently only available in larger stroke centres and can only be used in proximal vessel occlusion. In Germany, $7.7 \%$ of patients admitted for ischemic stroke received this treatment in 2019 (9). The more distal vessels or smaller vessels cannot be accessed by the thrombectomy catheters and can therefore not be recanalized by this approach, although the occlusion of those vessels can lead to detrimental effects for the patients, resulting in functional disability.

A promising approach to enhance recanalization with rt$\mathrm{PA}$ is the use of ultrasound (US). The first clinical report of stroke sonothrombolysis indicated increased rates of recanalization in patients receiving continuous transcranial Doppler ultrasound (TCD) monitoring during rt-PA therapy (14). Several small clinical trials with perflutrenlipid and galactose-based microbubbles as enhancers of sonothrombolysis suggest that microbubbles may produce further improvements in the rates of recanalization $(15-18)$. Despite the promise of this therapy, more than half of patients treated do not recanalize (19), and concerns have been raised regarding its safety with increased rates of haemorrhage in some studies $(17,20)$. The latest and largest trial in sonothrombolysis, the CLUTBUST-ER trial, demonstrated the safety of sonothrombolysis but failed to show beneficial effects on the clinical outcome (21). Therefore, there is still a great need for preclinical studies to better understand the efficacy and safety effects of this potential therapeutic strategy.

To test any thrombolytic therapy, a stroke model is required that uses a lifelike clot to block major cerebral arteries. Current thromboembolic models are highly variable, and it is likely that this variability is related to the choice of clot and its inherent stability with regards to spontaneous and thrombolytic-induced lysis (22).

In this article, we describe a method of embolic stroke using site-specific delivery of a whole blood thrombus to the origin of the middle cerebral artery (MCA). We aimed to investigate the effect of rt-PA therapy alone or in conjunction with US and a microbubble formulation (BR38; Bracco Suisse SA, Geneva, Switzerland) on recanalization rates in this model. BR38 is especially designed for usage in 
the therapy of acute intracranial artery occlusion and was described in detail by Schneider et al. (23).

We present the following article in accordance with the ARRIVE reporting checklist (available at http://dx.doi. org/10.21037/atm-21-75).

\section{Methods}

All experiments were performed in accordance with the German animal-protection legislation and were approved by the regional ethics committee of Regierungspraesidium Darmstadt (Az B 20/1120).

Sixty-seven male Wistar rats weighing $288 \pm 24.81 \mathrm{~g}$ were used in the present study (Envigo RMS GmbH, In den Leppsteinswiesen 19, 64380 Roßdorf, Germany \& Charles River, Sandhofer Weg 7, 97633 Sulzfeld, Germany).

The animals were anaesthetized with $5 \%$ isoflurane inhalation for 2 minutes. Anaesthesia was maintained with $2 \%$ to $3 \%$ isoflurane inhalation at $0.5 \mathrm{~L} / \mathrm{min}$ during surgery using a facial mask. For analgesia, the rats were treated with $0.05 \mathrm{mg} / \mathrm{kg}$ bodyweight buprenorphine (Buprenovet) 30 minutes prior to middle cerebral artery occlusion (MCAO) and in the evening after MCAO to ensure analgesia overnight. During surgery, $2 \%$ lidocaine was subcutaneously administered prior to cutting through the skin.

Body temperature was monitored and maintained at 36.5 to $37.0^{\circ} \mathrm{C}$. Dexpanthenol crème (Bepanthen, Bayer Vital) was used to prevent the eyes from drying out.

\section{Thrombus preparation}

The day before MCAO, the animals were anaesthetized, and blood was taken from the tail vein. Ten 15 -cm-long PE50 catheters were filled with blood, resulting in a total of $0.6 \mathrm{~mL}$. After incubation of the catheters at $37^{\circ} \mathrm{C}$ for 2 hours, a small part of the catheter was removed on both sides. Using a saline-filled syringe, the thrombi were flushed into a petri dish filled with saline. From the 10 resulting thrombi, a sufficiently long $(4 \mathrm{~cm})$ and uniformly thick and smooth thrombus was selected to be used for MCAO. The thrombus was then stored for 24 hours in a refrigerator at $4{ }^{\circ} \mathrm{C}$.

\section{MCAO}

Rats were subjected to MCAO using a thromboembolic model as previously described (24). In short, the rat was anaesthetized, and the right external carotid artery (ECA) and the right common carotid artery (CCA) were cauterized permanently, whereas a transient ligation of the internal carotid artery (ICA) was performed right after the bifurcation. A PE10 catheter was introduced through a small arteriotomy in the ECA. The catheter was advanced over the CCA in the ICA beyond the pterygopalatine artery until a mild resistance was met at the origin of the MCA. The PE10 catheter was then connected to a second PE10 catheter that contained the aforementioned thrombus. The rat was then turned on the belly, and, after incision and mobilization of the scalp, a laser Doppler probe (Oxford Optronix probe with Oxyflow 2000 Microvascular Perfusion Monitor) was positioned between the bregma and the lambda suture. After ensuring a stable signal from the laser Doppler, the thrombus was then flushed from the catheter using $0.1-\mu \mathrm{L}$ saline, resulting in a reduction of blood flow in the medial cerebral artery. A successful position of the thrombus resulted in a stable reduction of the blood flow to $50-90 \%$ from baseline. After waiting 40 minutes, treatment was started.

\section{Treatment}

For transcranial US application, the laser Doppler probe was removed, and the skin flaps of the scalp were held to the side using sutures. A 3-Mhz Doppler diagnostic US probe (Sonos 7500; Philips Ultrasound, USA; probe: diagnostic phased array probe with 80 elements) was placed above the skull, and the distance between the skull and ultrasonic probe was bridged with US gel (Transatlantic Handelsgesellschaft Stolpe \& Co. mbH, Neu-Anspach, DE). Transcranial colour-coded duplex ultrasound (TCCD) was applied continuously for the duration of the treatment (B-mode, colour Doppler functions switched on, approximate size of the colour box: $4.9 \mathrm{~cm} \times 8.1 \mathrm{~cm}$ ). The Doppler beam was aligned to expose the entire brain incorporating the circle of Willis and the occluded MCA. The spectral Doppler sample volume $(57 \mathrm{~mm})$ was placed in the midbrain [pulse duration $2 \mathrm{~ms}$, maximum output (mechanical index of the Doppler of 1.7)]. In rats without Doppler treatment (see below), the probe was placed, but the device remained deactivated.

The animals were randomized into four groups: rt-PA\& BR38-placebo without US (placebo), rt-PA-placebo \& BR38 with US, rt-PA \& BR-38 placebo without US (rt$\mathrm{PA}$ ), and rt-PA \& BR38 with US (US + rt-PA). BR38 and rt-PA were administered via the tail vein. rt-PA (Actilyse, 
Boehringer Ingelheim, Ingelheim, Germany) was dosed at $10 \mathrm{mg} / \mathrm{kg}$ bodyweight $(10 \%$ as bolus and $18 \%$ every 10 minutes). Four doses of $0.1-\mathrm{mL}$ BR38 $(10-\mu \mathrm{L}$ BR 38 diluted in $90-\mu \mathrm{L}$ saline; $4 \times 10^{8}$ bubbles $/ \mathrm{mL}$ ) were given at the start and every 15 minutes of treatment. The corresponding amount of saline served as a placebo. Treatment duration was 60 minutes total.

\section{Neurological examination and imaging}

Neurological evaluation was performed prior to anaesthesia and 24 hours after induction of ischemia. We applied a neurological score with 10 different sensorimotor and coordinative items, as described by Nedelmann et al. (25). Furthermore, animals were placed on a rotarod that was continuously accelerated from 0 to 30 rounds per minute (rpm). The maximum speed that the animals tolerated without falling off the device was recorded (26). The rotarod scores before surgery and 24 hours after surgery were subtracted to display the deterioration.

The animals were subjected to MRI imaging 90 minutes and 24 hours after MCAO. MRI measurements were performed using a 7 Tesla MRI spectrometer (PharmaScan; Bruker, Billerica, MA, USA) equipped with a $760-\mathrm{mT} / \mathrm{m}$ gradient system using a $20-\mathrm{mm}{ }^{1} \mathrm{H}$ receive-only surface coil together with a $72-\mathrm{mm}$ transmit-only volume resonator. For the MRI examination, the animals were fixed in a body restrainer with a tooth bar and a cone-shaped head. Respiratory rate was monitored by a pressure probe placed between the restrainer and the animal's thorax. Anaesthesia was maintained with isoflurane delivered through air at $0.6 \mathrm{~L} / \mathrm{min}$. The isoflurane concentration varied between $2.0 \%$ and $3.0 \%$ to keep the respiratory rate between 35 and $45 / \mathrm{min}$. Temperature was monitored using a rectal probe and maintained at $37{ }^{\circ} \mathrm{C}$ by a thermostatically regulated water flow system during the entire imaging protocol.

Localizer images were acquired using a spin-echo sequence. Rapid acquisition with refocusing echoes (RARE) sequences [20 contiguous slices, $1 \mathrm{~mm}$ thickness, repetition time $(\mathrm{TR})=2,500 \mathrm{~ms}$, echo time $(\mathrm{TE})=36.72 \mathrm{~ms}$ ] were used to verify symmetric positioning and were repeated after correction of slice angulation, if necessary.

A Carr-Purcell-Meiboom-Gill spin-echo imaging sequence was used to map lesion and hemisphere volumes. Twelve contiguous coronal slices with a thickness of $1 \mathrm{~mm}$ (gap $0 \mathrm{~mm}$ ) were acquired [field of view (FOV) $35 \times 35 \mathrm{~mm}$, matrix $512 \times 256$, TR $=3,800 \mathrm{msec}, 12$ echoes: $\mathrm{TE}=18 \mathrm{msec}$. Diffusion-weighted imaging was also used with a slice thickness of $1 \mathrm{~mm}$ (FOV $35 \times 35 \mathrm{~mm}$, matrix $128 \times 128$, TR $=3,000 \mathrm{~ms}$, TE $=43 \mathrm{~ms}$ ). Susceptibility-weighted sequences (SWI) with a slice thickness of $1 \mathrm{~mm}$ were used to detect bleeding complications (FOV $35 \times 35 \mathrm{~mm}$, matrix $384 \times 384$, $\mathrm{TR}=720 \mathrm{~ms}$, TE $=18 \mathrm{~ms}$ ).

Infarct size and hemispheric volume were measured with planimetry in T2-weighted and diffusion-weighted sequences (Phillips Dicom Viewer 2.0). The SWI sequences were checked for the presence of intracerebral haemorrhage. Computer-aided planimetric assessments of ischemic lesion volumes and hemispheric volumes were performed by two blinded investigators experienced in experimental stroke MRI and sufficient training in planimetry (27). After optimal adjustment of contrast, the edges of the hemispheres were traced manually on each slice using neuroanatomic landmarks. The edges of the hyperintense ischemic lesions were traced manually in a similar fashion. The areas were then summed and multiplied by the slice thickness to calculate volumes. Lesion volumes were also expressed as a percent of the hemispheric volume.

\section{Decapitation and histological staining}

The animals were decapitated while under deep anaesthesia after completing the second MRI, and then the brains were quickly removed from the skull and inspected to detect side effects such as subarachnoid haemorrhage. The brains were fixed in a $4 \%$ formaldehyde solution (Roti ${ }^{\circledR}$ Histofix). After slicing and preparation, the slices were stained using haematoxylin and eosin. The slices were examined under the microscope and evaluated for microbleedings using a software (ZEN-Blue 2.6 by Zeiss).

\section{Exclusion criteria}

The animals were excluded in the case of severe dyspnoea and neurological impairment that made the ingestion of food and water impossible. If the MRI showed no infarction in the ipsilateral hemisphere, infarction in the contralateral hemisphere, or just a small infarction volume $\left(<0.1 \mathrm{~cm}^{3}\right)$, the animals were excluded because of model failure.

\section{Statistical analysis}

Data are presented as mean \pm standard deviation. All data was tested for normal distribution and variance homogeneity. Functional Assessment was tested using a $t$-test. The difference in the groups in terms of bleeding 
Table 1 Distribution of surviving animals in the different treatment groups

\begin{tabular}{lc}
\hline Group & Number of surviving animals \\
\hline Placebo & 3 \\
US & 6 \\
rt-PA & 6 \\
US + rt-PA & 5 \\
\hline
\end{tabular}

US, ultrasound; rt-PA, recombinant tissue plasminogen activator.

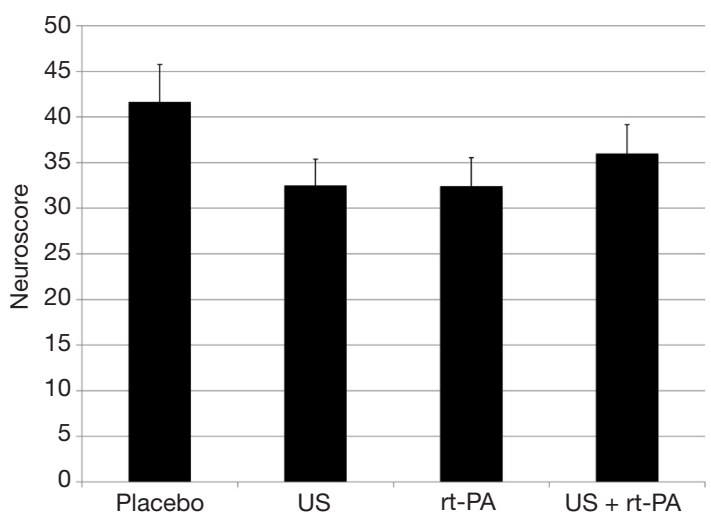

Figure 1 Mean value with standard deviation of the NeuroScore 24 hours after the thrombus induction. There is no significant difference between the four treatment groups $(\mathrm{P}>0.05)$. US, ultrasound; rt-PA, recombinant tissue plasminogen activator.

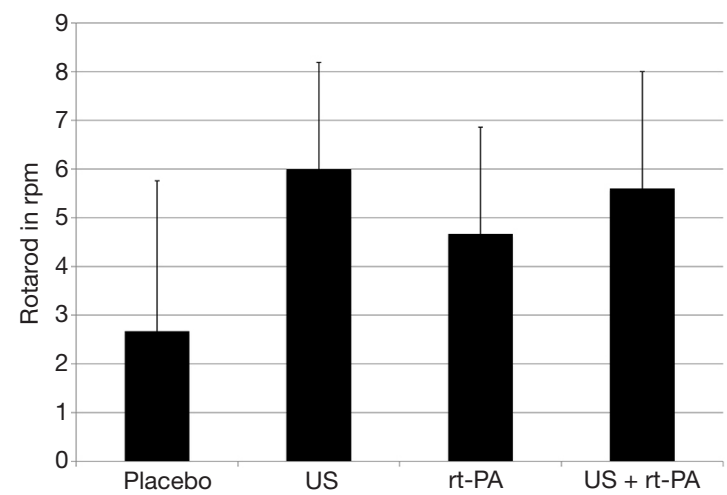

Figure 2 Mean value with standard deviation of the rotarod test in rpm. The values show the difference between the first (preoperative) and second (postoperative) point of measurement. There is no significant difference between the four treatment groups $(\mathrm{P}>0.05)$. rpm, rounds per minute. US, ultrasound; rt-PA, recombinant tissue plasminogen activator. frequencies and Mortality was tested with an exact Fishertest. Group differences were tested using a mixed-model analysis (MMA). A $P$ value $\leq 0.05$ was considered statistically significant (SPSS v22, IBM, Germany).

\section{Results}

Only 20 (29.9\%) animals completed the experiment and were analysed per protocol. Twelve animals died during the surgery and the treatment procedure (17.9\%). Another 10 animals died over the course of the night after the surgery $(14.9 \%)$. Five animals $(7.5 \%)$ had to be excluded due to dyspnoea or disabling neurological impairment. The MRI examination detected model failure in 20 (29.9\%) animals.

For the distribution of the surviving animals, see Table 1.

The physiological blood parameters, body temperature, and bodyweight of the remaining animals did not differ between the experimental groups and remained within the normal physiological range throughout the surgical and treatment procedure.

All surviving animals displayed clinical signs of MCA territory stroke 24 hours after the procedure. Clinical evaluation using the NeuroScore revealed no differences between groups (placebo 41.67 \pm 4.07 ; US $32.5 \pm 2.88$; rtPA 32.43 \pm 3.12 ; US + rt-PA 36 \pm 3.15 ; P>0.05; MMA; Figure 1). Rotarod testing also showed no differences between the groups (placebo $2.67 \pm 3.09 \mathrm{rpm}$; US $6 \pm 2.19$ rpm; rt-PA 4.67 \pm 2.19 rpm; US + rt-PA 5.6 \pm 2.4 rpm; P>0.05; MMA; Figure 2).

The absolute infarct size did not differ between the groups 90 minutes after surgery (placebo $0.08 \pm 0.10 \mathrm{~cm}^{3}$; US $0.09 \pm 0.07 \mathrm{~cm}^{3} ; \mathrm{rt}-\mathrm{PA} \quad 0.16 \pm 0.07 \mathrm{~cm}^{3}$; US + rt-PA $0.10 \pm 0.08 \mathrm{~cm}^{3} ; \mathrm{P}>0.05 ;$ MMA; Figure 3). There were also no differences when calculating the percentual infarct size of the affected hemisphere (placebo $6.86 \% \pm 9.09 \%$; US 7.61\% $\pm 6.43 \%$; rt-PA $14.61 \% \pm 6.43 \%$; US + rt-PA $9.45 \% \pm 7.04 \%$; P>0.05; MMA; Figure 4). Figure 5 shows exemplary $\mathrm{T} 2$-weighted images of the different groups.

Twenty-four hours after surgery, the placebo group had the largest ischemic volumes as compared to the treatment groups. Those differences were statistically significant. The lesional volume was smallest in the combined treatment group (US + rt-PA). However, there were no statistical differences when comparing the treatment groups, but all treatment groups were significantly smaller compared to Placebo (placebo $0.75 \pm 0.10 \mathrm{~cm}^{3}$; US $0.43 \pm 0.07 \mathrm{~cm}^{3}$; rt-PA 


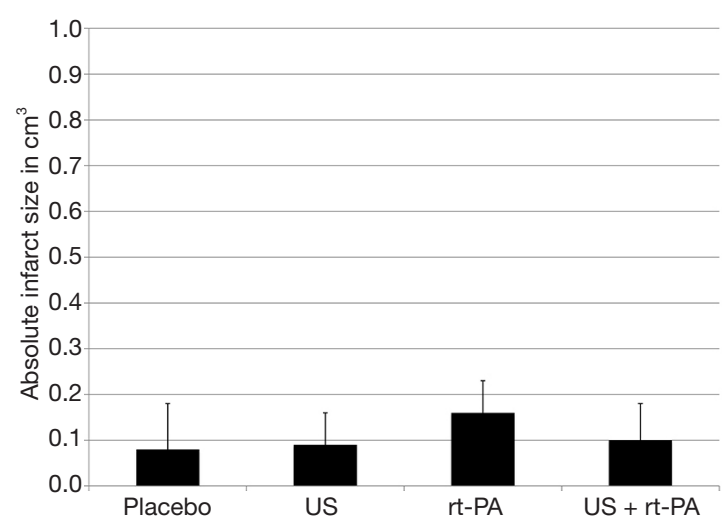

Figure 3 Mean value with standard deviation of the absolute infarct size in $\mathrm{cm} 390$ minutes after thrombus induction. There is no significant difference between the four treatment groups $(\mathrm{P}>0.05)$. US, ultrasound; rt-PA, recombinant tissue plasminogen activator.

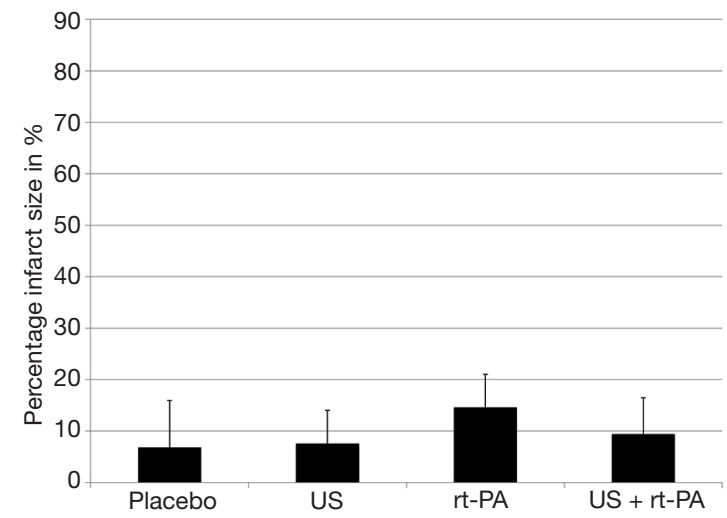

Figure 4 Mean value with standard deviation of the infarct size as percentage of ipsilateral hemispheric volume 90 minutes after thrombus induction. There is no significant difference between the four treatment groups $(\mathrm{P}>0.05)$. US, ultrasound; rt-PA, recombinant tissue plasminogen activator.

$0.4 \pm 0.07 \mathrm{~cm}^{3} ;$ US + rt-PA $0.27 \pm 0.08 \mathrm{~cm}^{3} ; \mathrm{P}>0.05 ;$ MMA; Figure 6). The same result was also found when calculating the infarct size as percentage of the ipsilateral hemispheric volume (placebo $67.78 \% \pm 9.09 \%$; US $37.76 \% \pm 6.43 \%$; rtPA $23.96 \% \pm 7.04 \%$; US + rt-PA $34.62 \% \pm 6.43 \%$; P>0.05; MMA; Figure 7).

The absolute differences of ischemic volume 90 minutes and 24 hours after surgery were compared to visualize infarct growth. The largest growth was found in the placebo group $\left(0.67 \mathrm{~cm}^{3} ; \mathrm{P}<0.001 ; \mathrm{MMA}\right)$. There was also a statistically significant growth in the US group $\left(0.34 \mathrm{~cm}^{3}\right.$; $\mathrm{P}<0.001 ; \mathrm{MMA})$ and the rt-PA group $\left(0.24 \mathrm{~cm}^{3} ; \mathrm{P}<0.007\right.$; MMA). The US + rt-PA group showed the smallest growth that missed the significant level $\left(0.17 \mathrm{~cm}^{3} ; \mathrm{P}<0.062 ; \mathrm{MMA}\right)$.

Intracerebral bleeding was found using MRI imaging in 11 animals 24 hours after surgery. There were no differences between the groups [placebo 3 (100\%); US 4 (67\%); rt-PA 2 (33\%); US + rt-PA 2 (40\%); P>0.05; Fisher-test]. Clinical deficits and functional performance did not differ in animals with and without intracerebral bleeding in MRI imaging (NeuroScore: bleeding $37.27 \pm 8.17$, no bleeding $31.25 \pm 7.91$, $\mathrm{P}>0.05, t$-test; rotarod: bleeding $3.27 \pm 4.92$, no bleeding $7.11 \pm 6.79, \mathrm{P}>0.05, t$-test). Histological staining revealed at least small intracerebral bleedings in all animals.

Of the 22 animals that died during surgery, 8 were in the placebo group (36.4\%), 2 were in the US group (9.1\%), 7 were in the rt-PA group (31.8\%), and 5 were in the US + rtPA group (22.7\%). There were no statistical differences in the mortality between the groups $(\mathrm{P}>0.05$; Fisher-test).

\section{Discussion}

Our study showed a lower lesional volume in all animals with active therapy as compared to the placebo. There were no differences between the different treatment groups.

The safety evaluation of the different treatments using histological techniques revealed microbleedings in all animals, even in the placebo group. There were no statistical differences in the frequency of macroscopic intracerebral haemorrhage, detected by SWI sequences, or in mortality. Clinical performance of the animals with intracerebral haemorrhage did not differ from animals without intracerebral haemorrhage. As we saw no differences of intracerebral haemorrhage between the groups a delayed recanalization seems unlikely as a mechanism of action for sonothrombolysis. A delayed recanalization is associated with reperfusion injury and a higher rate of intracerebral haemorrhage (28).

Ninety minutes after thrombus induction, we saw no differences between the treatment groups. This might be mainly due to the circumstance, that T2-imaging, that reflects cerebral vasogenic oedema, needs time to show an ischemic lesion. Ninety minutes after thrombus induction, there might remain some penumbral tissue that is still vital via collaterals that deteriorate over the course of time, resulting in infarct growth. The vasogenic oedema leads to secondary infarct growth due to compressive effects on cerebral vessels, but needs time to develop. It is responsible 

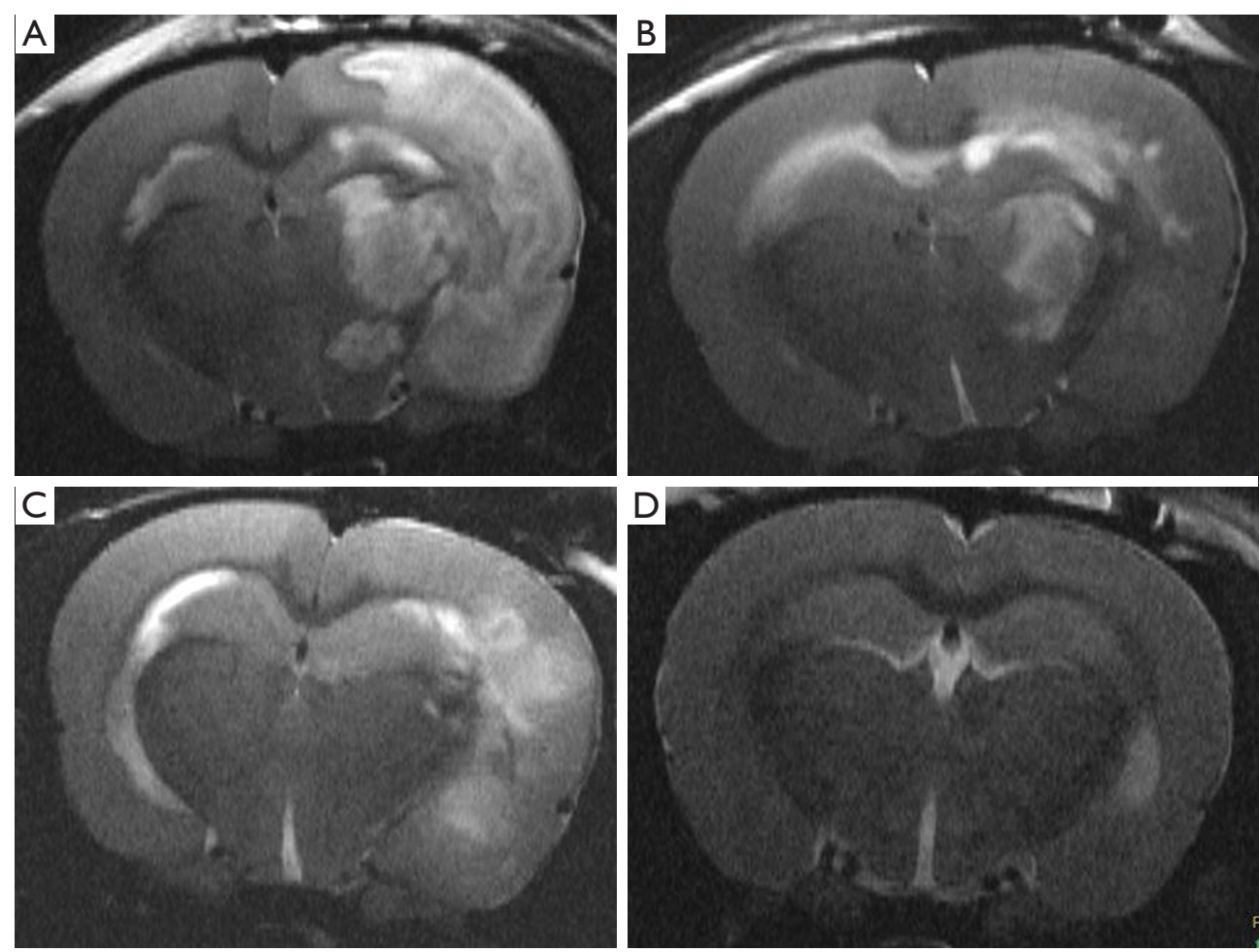

Figure 5 Examples of T2-weighted images of the infarct volume for the four different treatment groups. (A) Placebo; (B) US; (C) rt-PA; (D) US + rt-PA. US, ultrasound; rt-PA, recombinant tissue plasminogen activator.

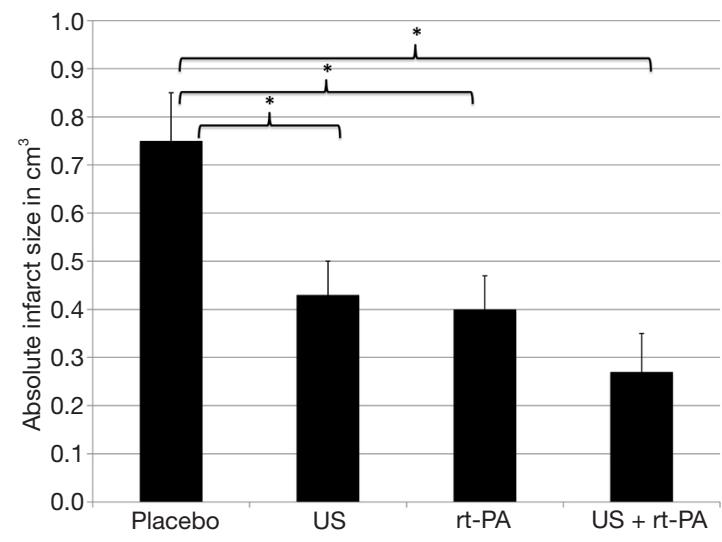

Figure 6 Mean value with standard deviation of the absolute infarct size in $\mathrm{cm} 324$ hours after thrombus induction. There is a significant difference between Placebo and US ( $\mathrm{P}=0.016)$, Placebo and rt-PA $(\mathrm{P}=0.09)$, and Placebo and $\mathrm{US}+\mathrm{rt}-\mathrm{PA}(\mathrm{P}=0.001)$. Asterisks are used to mark $\mathrm{P}$ values below 0.05 . US, ultrasound; rt$\mathrm{PA}$, recombinant tissue plasminogen activator.

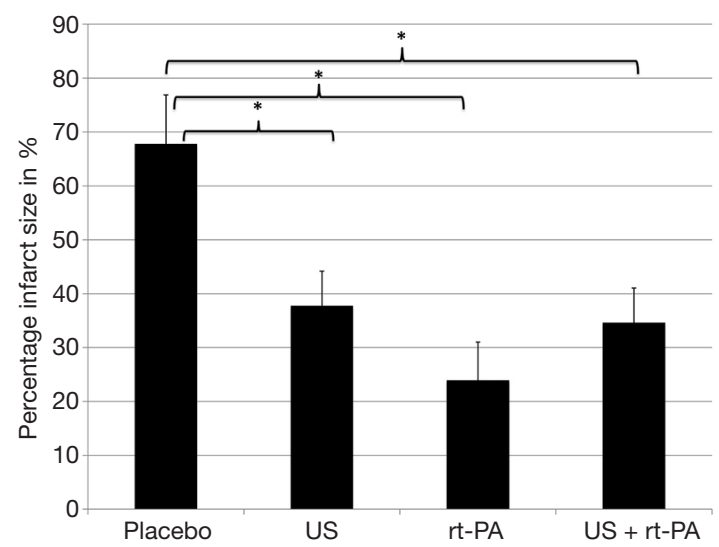

Figure 7 Mean value with standard deviation of the infarct size as percentage of ipsilateral hemispheric volume 24 hours after thrombus induction. There is a significant difference between Placebo and US $(\mathrm{P}=0.012)$, Placebo and rt-PA $(\mathrm{P}=0.006)$, and Placebo and US + rt-PA $(\mathrm{P}=0.001)$. Asterisks are used to mark $\mathrm{P}$ values below 0.05 . US, ultrasound; rt-PA, recombinant tissue plasminogen activator. 
for up to $50 \%$ of the definite infarct volume (29).

Regarding the safety of microbubble-mediated sonothrombolysis (mmSTL), no existing experimental in vivo study revealed a higher rate of cerebral haemorrhage, irrespective of stroke model, animal, microbubble use, or use of a concomitant thrombolytic agent (22). In most studies, mmSTL was classified as efficient. However, numerous primary endpoints were used such as lesional volume, presence of recanalization, declotting time, D-Dimer values, and clinical scores. Further methodological differences include the animal model (swine, rat, rabbit), clot preparation (autologous, human clots, red or white clots), route of microbubble administration (arterial or venous), US parameters, and use of rt-PA (22). This heterogeneity complicates the classification of our results.

Only eight studies examined mmSTL in rats (30-37). In six of those studies, mmSTL was combined with rt-PA (30-34,37).

Nedelmann et al. and Schleicher et al. used a filament model to assess microvascular impairment. Nedelmann et al. demonstrated less vascular impairment of the ipsilateral hemisphere after 60 minutes of transient MCAO when using mmSTL in combination with rt-PA as compared to the placebo, rt-PA alone, mmSTL alone, or the combination of rt-PA and sonothrombolysis without microbubbles. They also showed a lower infarct volume and less cerebral oedema but no differences in clinical outcome in $\mathrm{mmSTL}$ in combination with rt-PA as compared to rt-PA alone (33). Schleicher et al. examined rt-PA with mmSTL with two different microbubble types (Sonovue ${ }^{\circledR}$ and BR $38^{\circledR}$ ) and different microbubble dosages (full dose vs. $1 / 3$ dose). They were able to demonstrate less vascular impairment of the ipsilateral hemisphere in all mmSTLtreated animals as compared to the controls or rt-PA alone. They also examined ischemic lesion volume histologically in controls, rt-PA only, and the combination of rt-PA and mmSTL (BR38 ${ }^{\circledR}$; full dosage). The combination of both therapies showed less ischemic volume as compared to the controls (31). Ren et al. and Lu et al. used fragmented thrombi to induce ischemia. When using a whole blood thrombus and Sonovue ${ }^{\circledR}$, Ren et al. were able to show a faster recanalization when combining mmSTL with rtPA after 10 minutes of treatment. However, rt-PA and a combination of mmSTL and rt-PA in halved dosages showed these results after 20 minutes of treatment. Twenty minutes after ischemia induction, mmSTL alone did not result in recanalization (32). Lu et al. used white and red thrombi in combination with a placebo, sonothrombolysis without microbubbles, mmSTL alone, rt-PA alone, or rt-PA combined with mmSTL. As a microbubble type, lipid shelled perfluoropropane microbubbles were used. mmSTL reduced the infarct volume and showed a better clinical outcome irrespective of the used thrombi. mmSTL led to a lower infarct volume when using red thrombi compared to rt-PA, whereas there was a better clinical outcome in mmSTL and white thrombi compared to rtPA. The combination of rt-PA with mmSTL led to a lower infarct volume when using red thrombi as compared to rtPA alone. This effect was not reported when using white thrombi (34). Tomkins et al. used a continuous 3-cm-long white clot thrombus to achieve MCAO and BR38 as the microbubble type. The white clot proved to be resistant to the effects of mmSTL combined with rt-PA in this model (30).

Dixon et al. used a microfluidic device to produce microbubbles. MCAO was achieved by a continuous full blood thrombus. Microbubbles were directly administered into the ICA. They compared mmSTL with low-dose rt$\mathrm{PA}$ to low-dose rt-PA alone, high-dose rt-PA alone, and controls. Ischemic volume was lower, and clinical outcome was better in high-dose rt-PA-treated animals as compared to the controls. There was no reduction in infarct volume in mmSTL with rt-PA as compared to the controls, but there was a better clinical outcome 24 hours after treatment (37).

Our study showed efficacy and safety of mmSTL with or without t-PA in an embolic rat stroke model using a continuous full blood thrombus. It is difficult to compare our results to Dixon et al. They reported a clot volume of $16 \mu \mathrm{L}$, administered via a PE10 catheter. The diameter of the PE-10 catheter was not specified and varies among different manufacturers (37). When calculating the length using an inner diameter of $0.28 \mathrm{~mm}$, and if all of this volume consisted of thrombus material, this would result in a 26-cm-long thrombus administered to the rat. We were able to show an effect of mmSTL without use of rt-PA. $\mathrm{Lu}$ et al. and Ren et al. demonstrated an effect of mmSTL without rt-PA in rats, but used a different clot model $(32,34)$. Brown et al. were also able to show an effect of mmSTL alone, but in rabbits (38). In vitro-studies have suggested, that moderate US intensities have a limited effect on thrombolysis without use of rt-PA (39). However, in the in vivo situation, intrinsic t-PA is present that might facilitate a thrombolytic effect.

Our experiment underlines the efficacy and safety of mmSTL in experimental in vivo stroke models. Nevertheless, in human stroke studies, a meta-analysis 
of sonothrombolysis with or without microbubble use showed no beneficial effects $(16,17,40)$. Different contrast enhancers, such as Levovist ${ }^{\circledR}$, Sonovue ${ }^{\circledR}$, or microspheres, were used in the trials. In the largest trial, the CLOTBUSTER trial, there was no use of a contrast enhancer (21). The use of mmSTL is partially limited by the administration of microbubbles, as they must be given either continually or with multiple boli over the course of the therapy. The relevance of sonothrombolysis in a clinical setting remains debatable, especially since the endovascular therapy studies demonstrated their large treatment effects. As Alexandrov et al. noted, new trials testing new non-endovascular approaches will be difficult to realize in the future, and it is questionable if new studies for sonothrombolysis will arise (41). Using a user independent head mount for insonation, the latest trial, the TRUST study, has not been recruiting since 2018 (source: clinicaltrials.gov/ct2/show/ NCT03519737), but no results have yet been published. It remains questionable if sonothrombolysis has a role in cerebral large vessel occlusion, as those patients should undergo endovascular therapy. A scenario, in which a patient would not be eligible for this therapy, does not seem to be realistic. However, sonothrombolysis could be used during the transport from a primary stroke centre to a hospital for endovascular therapy. This setting was addressed in the TRUST trial. Patients with more distal vessel occlusion could also benefit from the effects of sonothrombolysis. Nevertheless, sonothrombolysis might share the fate of neuroprotection, as it might be effective in animal models but shows no effect on functional outcome of stroke patients. Therefore, sonothrombolysis will still play no role in clinical routine.

Our study is limited by the small number of animals completing the study protocol. However, we were able to show statistical differences regarding the efficacy of the treatment. There might be differences in the safety of the treatment that might not have been detected. As most animals died over the course of the night, we were unable to obtain direct information about the cause of death. Therefore, our study is also limited by the inability to differentiate if mortality resulted from the stroke model or as a side effect of the treatment. The short observation period after a stroke (24 hours) also limits our study because, at 24 hours after ischemia, the lesional size might not have reached its maximum. The outcome might not be stable, and late deficits could appear over the course of time (42). A longer observational period would not have been approved by our local animal ethics committee.
To improve the model, it should be possible to produce a thrombus that always has the same constitutions and comparable properties to humans. Thus, the scatter of infarct sizes would no longer exist and the nonresponsive or dead animals can be eliminated in advance. The intraoperative mortality might be lower when using continuous pulse oximetry and orotracheal intubation. By this approach, lower dosages of isoflurane can be used which might lead to a lower rate of complications and the person performing the surgery can react faster to changes in $\mathrm{SpO}_{2}$ and the heart rate. By this approach, mortality and rate of model failure might be reduced.

\section{Conclusions}

Our study showed the efficacy and safety of mmSTL with or without rt-PA in an embolic rat stroke model using a continuous full blood thrombus. Sonothrombolysis might be useful for patients who need to be transported to a thrombectomy centre or for those with distal vessel occlusion.

\section{Acknowledgments}

Funding: This research was supported by a research grant of the University Medical Center Giessen and Marburg (UKGM) [5/2016 GI] and by Bracco Suisse SA, Geneva, Switzerland.

\section{Footnote}

Reporting Checklist: The authors have completed the ARRIVE reporting checklist. Available at http://dx.doi. org/10.21037/atm-21-75

Data Sharing Statement: Available at http://dx.doi. org/10.21037/atm-21-75

Peer Review File: Available at http://dx.doi.org/10.21037/ atm-21-75

Conflicts of Interest: All authors have completed the ICMJE uniform disclosure form (available at http://dx.doi. org/10.21037/atm-21-75). TB reports grants from the University Medical Center Giessen and Marburg (UKGM), Germany; non-financial support from Bracco Suisse SA, Geneva, Switzerland, during the conduct of the study; nonfinancial support from Bayer Vital GmbH, outside the 
submitted work. The other authors have no conflicts of interest to declare.

Ethical Statement: The authors are accountable for all aspects of the work in ensuring that questions related to the accuracy or integrity of any part of the work are appropriately investigated and resolved. Experiments were performed under a project license (No. Az B 20/1120) granted by the regional ethics committee of Regierungspraesidium Darmstadt, in compliance with German national guidelines for the care and use of animals.

Open Access Statement: This is an Open Access article distributed in accordance with the Creative Commons Attribution-NonCommercial-NoDerivs 4.0 International License (CC BY-NC-ND 4.0), which permits the noncommercial replication and distribution of the article with the strict proviso that no changes or edits are made and the original work is properly cited (including links to both the formal publication through the relevant DOI and the license). See: https://creativecommons.org/licenses/by-nc-nd/4.0/.

\section{References}

1. Benjamin EJ, Blaha MJ, Chiuve SE, et al. Heart disease and stroke statistics-2017 update: a report from the American Heart Association. Circulation 2017;135:e146603. Erratum in: Circulation 2017;135:e646. Erratum in: Circulation 2017;136:e196.

2. Mathers CD, Boerma T, Ma Fat D. Global and regional causes of death. Br Med Bull 2009;92:7-32.

3. Ntaios G, Hart R. Embolic stroke. Circulation 2017;136:2403-5.

4. Hacke W, Kaste M, Bluhmki E, et al. Thrombolysis with alteplase 3 to 4.5 hours after acute ischemic stroke. $\mathrm{N} \mathrm{Engl}$ J Med 2008;359:1317-29.

5. Goyal M, Demchuk A, Menon B, et al. Randomized assessment of rapid endovascular treatment of ischemic stroke. N Engl J Med 2015;372:1019-30.

6. Ma H, Campbell B, Parsons M, et al. Thrombolysis guided by perfusion imaging up to 9 hours after onset of stroke. $\mathrm{N}$ Engl J Med 2019;380:1795-803.

7. Nogueira RG, Jadhav AP, Haussen DC, et al. Thrombectomy 6 to 24 hours after stroke with a mismatch between deficit and infarct. N Engl J Med 2018;378:11-21.

8. Thomalla G, Simonsen C, Boutitie F, et al. MRI-guided thrombolysis for stroke with unknown time of onset. $\mathrm{N}$ Engl J Med 2018;379:611-22.
9. Richter D, Eyding J, Weber R, et al. Analysis of nationwide stroke patient care in times of COVID-19 pandemic in Germany. Stroke 2021;52:716-21.

10. Rha JH, Saver J. The impact of recanalization on ischemic stroke outcome: a meta-analysis. Stroke 2007;38:967-73.

11. Tsivgoulis G, Katsanos A, Schellinger P, et al. Successful reperfusion with intravenous thrombolysis preceding mechanical thrombectomy in large-vessel occlusions. Stroke 2018;49:232-5.

12. Rabinstein AA. Treatment of acute ischemic stroke. Continuum (Minneap Minn) 2017;23:62-81.

13. Balami JS, Sutherland BA, Edmunds LD, et al. A systematic review and meta-analysis of randomized controlled trials of endovascular thrombectomy compared with best medical treatment for acute ischemic stroke. Int J Stroke 2015;10:1168-78. Erratum in: Int J Stroke 2017;12:NP7.

14. Alexandrov AV, Demchuk AM, Felberg RA, et al. High rate of complete recanalization and dramatic clinical recovery during tPA infusion when continuously monitored with 2-MHz transcranial doppler monitoring. Stroke 2000;31:610-4.

15. Alexandrov AV, Mikulik R, Ribo M, et al. A pilot randomized clinical safety study of sonothrombolysis augmentation with ultrasound-activated perflutrenlipid microspheres for acute ischemic stroke. Stroke 2008;39:1464-9.

16. Molina CA, Barreto AD, Tsivgoulis G, et al. Transcranial ultrasound in clinical sonothrombolysis (TUCSON) trial. Ann Neurol 2009;66:28-38.

17. Molina CA, Ribo M, Rubiera M, et al. Microbubble administration accelerates clot lysis during continuous 2-MHz ultrasound monitoring in stroke patients treated with intravenous tissue plasminogen activator. Stroke 2006;37:425-9.

18. Viguier A, Petit R, Rigal M, et al. Continuous monitoring of middle cerebral artery recanalization with transcranial color-coded sonography and Levovist. J Thromb Thrombolysis 2005;19:55-9.

19. Alexandrov AV, Molina CA, Grotta JC, et al. Ultrasoundenhanced systemic thrombolysis for acute ischemic stroke. N Engl J Med 2004;351:2170-8.

20. Daffertshofer M, Gass A, Ringleb P, et al. Transcranial low-frequency ultrasound-mediated thrombolysis in brain ischemia: increased risk of hemorrhage with combined ultrasound and tissue plasminogen activator: results of a phase II clinical trial. Stroke 2005;36:1441-6.

21. Alexandrov AV, Köhrmann M, Soinne L, et al. Safety and 
efficacy of sonothrombolysis for acute ischaemic stroke: a multicentre, double-blind, phase 3, randomised controlled trial. Lancet Neurol 2019;18:338-47.

22. Auboire L, Sennoga C, Hyvelin JM et al. Microbubbles combined with ultrasound therapy in ischemic stroke: a systematic review of in-vivo preclinical studies. PLoS One 2018;13:e0191788.

23. Schneider M, Anantharam B, Arditi M, et al. BR38, a new ultrasound blood pool agent. Invest Radiol 2011;46:486-94.

24. Zhang RL, Chopp M, Zhang ZG, et al. A rat model of focal embolic cerebral ischemia. Brain Res 1997;766:83-92.

25. Nedelmann M, Wilhelm-Schwenkmezger T, Alessandri $\mathrm{B}$, et al. Cerebral embolic ischemia in rats: correlation of stroke severity and functional deficit as important outcome parameter. Brain Res 2007;1130:188-96.

26. Hamm RJ, Pike BR, O'Dell DM, et al. The rotarod test: an evaluation of its effectiveness in assessing motor deficits following traumatic brain injury. J Neurotrauma 1994;11:187-96.

27. Braun T, Pukropski J, Yeniguen M, et al. Inter- and intra-rater reliability of computer-assisted planimetry in experimental stroke research. J Neurosci Methods 2019;312:12-5.

28. Molina CA, Montaner J, Abilleira S, et al. Timing of spontaneous recanalization and risk of hemorrhagic transformation in acute cardioembolic stroke. Stroke 2001;32:1079-84.

29. Walberer M, Ritschel N, Nedelmann M, et al. Aggravation of infarct formation by brain swelling in a large territorial stroke: a target for neuroprotection? J Neurosurg 2008;109:287-93.

30. Tomkins AJ, Schleicher N, Murtha L, et al. Platelet rich clots are resistant to lysis by thrombolytic therapy in a rat model of embolic stroke. Exp Transl Stroke Med 2015;7:2.

31. Schleicher N, Tomkins A, Kampschulte M, et al. Sonothrombolysis with BR38 microbubbles improves microvascular patency in a rat model of stroke. PLoS One 2016;11:e0152898.

32. Ren X, Wang Y, Wang Y, et al. Thrombolytic therapy with rt-PA and transcranial color Doppler ultrasound (TCCS) combined with microbubbles for embolic thrombus. Thromb Res 2015;136:1027-32.

33. Nedelmann M, Ritschel N, Doenges S, et al. Combined contrast-enhanced ultrasound and rt-PA treatment is safe and improves impaired microcirculation after reperfusion of middle cerebral artery occlusion. J Cereb Blood Flow
Metab 2010;30:1712-20.

34. Lu Y, Wang J, Huang R, et al. Microbubble-mediated sonothrombolysis improves outcome after thrombotic microembolism-induced acute ischemic stroke. Stroke 2016;47:1344-53.

35. Alonso A, Dempfle CE, Della Martina A, et al. In vivo clot lysis of human thrombus with intravenous abciximab immunobubbles and ultrasound. Thromb Res 2009;124:70-4.

36. Moumouh A, Barentin L, Tranquart F, et al. Fibrinolytic effects of transparietal ultrasound associated with intravenous infusion of an ultrasound contrast agent: study of a rat model of acute cerebral stroke. Ultrasound Med Biol 2010;36:51-7.

37. Dixon AJ, Li J, Rickel JR, et al. Efficacy of sonothrombolysis using microbubbles produced by a catheter-based microfluidic device in a rat model of ischemic stroke. Ann Biomed Eng 2019;47:1012-22.

38. Brown AT, Flores R, Hamilton E, et al. Microbubbles improve sonothrombolysis in vitro and decrease hemorrhage in vivo in a rabbit stroke model. Invest Radiol 2011;46:202-7.

39. Bader KB, Gruber MJ, Holland CK. Shaken and stirred: mechanisms of ultrasound-enhanced thrombolysis. Ultrasound Med Biol 2015;41:187-96. Erratum in: Ultrasound Med Biol 2019;45:2266.

40. Nacu A, Kvistad C, Naess H, et al. NOR-SASS (Norwegian Sonothrombolysis in Acute Stroke Study): randomized controlled contrast-enhanced sonothrombolysis in an unselected acute ischemic stroke population. Stroke 2017;48:335-41.

41. Alexandrov AV, Tsivgoulis G, Köhrmann M, et al. Endovascular equipoise shift in a phase III randomized clinical trial of sonothrombolysis for acute ischemic stroke. Ther Adv Neurol Disord 2019;12:1756286419860652.

42. Kahle MP, Bix GJ. Successfully climbing the "STAIRs": surmounting failed translation of experimental ischemic stroke treatments. Stroke Res Treat 2012;2012:374098.

Cite this article as: Braun T, Sünner L, Hachenberger $M$, Müller C, Wietelmann A, Juenemann M, Pons-Kühnemann J, Kaps M, Gerriets T, Tschernatsch M, Roth J, Yenigün M. Microbubble-mediated sonothrombolysis with BR38 of a venous full blood thrombus in a rat embolic stroke model. Ann Transl Med 2021;9(13):1061. doi: 10.21037/atm-21-75 